\section{Elastic compression treatment of chronic superficial venous insufficiency of the lower limbs based on Doppler venous pressure index measurements}

\author{
Leonardo Corcos,' Daniele Pontello, ${ }^{2}$ \\ Tommaso Spina $^{3}$ \\ ${ }^{1}$ Vascular Laboratory, Prosperius \\ Institute, Firenze; ${ }^{2} \mathrm{~S}$. Maria Maddalena \\ Private Hospital, Rovigo; ${ }^{3}$ ASP Cosenza, \\ Italy
}

\begin{abstract}
Ineffectiveness or discomfort from graduated elastic compression stockings (GES) in patients with chronic venous insufficiency (CVI) and/or varicose veins of the lower limbs (VVLL) can depend of inappropriate counter pressure applied. Counter pressure was calculated by Doppler venous pressure index (VPI). The aim of this study was to verify the value VPI in the choice of GES. A total of 1212 LL of 606 patients subjected to VPI measurements VPI correlated with the various sites of reflux (R) and $C$ of Clinical-Etiology-Anatomy-Pathophysiology (CEAP) classification. The difference between standing VPI the and normal values=counter pressure to be applied by GES. Questionnaire to 96 patients with CVI/WVLL wearing GES. Mean VPI values: greater saphenous (GSV)>smaller saphenous; GSV with isolated venous reflux (R) at the leg $>\mathrm{GSV}$ at the thigh; additional $\mathrm{R}$ in perforators increases VPI in all the districts; superficial R increases VPI in PT. Relation between VPI/C of CEAP: $\mathrm{P}<0.05-0.0001 ; 81 / 83 / 96$ (97.5\%) patients improved; 0 complained. $\mathrm{R}$ in GSV at the leg and in perforators increases VPI in deep veins. Few discrepancies VPI/CEAP can be expected. Standing VPI is highly predictive. The best choice of GES can be based on the VPI measurement.
\end{abstract}

\section{Introduction}

Elastic compression treatment (ECT) of chronic venous insufficiency (CVI) and varicose veins of the lower limbs (VVLL) by the application of various kinds of bandages and graduated elastic stockings (GES) has been strongly recommended in the past for conservative and invasive treatments of almost every phebological condition and still is being evolved by more recent studies. ${ }^{1-9}$ The stockings were divided in classes of various pressure degrees, from prevention GES up to the $4^{\circ}$ class $^{2-4}$ and the indications for the class of compression were mainly based on clinical criteria, especially in the treatment of chronic venous ulcers; the related positive results were clearly demonstrated by clinical and instrumental investigations. ${ }^{5,6}$ However, as it is well known, many patients complain with discomfort and pain by GES and they often refuse the treatment. The more frequent explanation are obesity, skeletal and joints alterations, kind of work, physical behavior, intolerance to the GES tissues and mainly to a wrong size of GES. In spite of more accuracy in measuring all the clinical and physical parameters recommended for the appropriate choice of the GES class of compression still some patients complain with pain or, at the opposite, do not improve their clinical condition. The problem is still unclear and the debated is still open. ${ }^{7-9}$

Therefore we supposed that the main factor influencing the clinical results from GES could be represented by a wrong counter pressure applied which could not be able to improve the subjective symptoms when too low or, at the opposite, being a main cause of discomfort and pain when too high. The only way for detecting the appropriate counter pressure to be applied seems to be the venous pressure measurement (VPM) of the affected limbs and the comparison with mean normal values.

Invasive VPM belong to the past of Phlebology ${ }_{10}^{10}$ and were more recently, ${ }^{11,12}$ nor could be actually proposed to the patients. The non invasive Doppler method described by Gayliss and Bartolo ${ }^{13,14}$ was statistically validated in $1983^{15}$ and it seems to represent the more acceptable procedure in the daily practice.

Since the 1985 we systematically measured the venous pressure index (VPI) by noninvasive Doppler method and compared the exceeding VPI, measured in standing position and after 10 tip-toeing exercises (ambulatory) of patients affected with venous disease in both the limbs, with the mean VPI detected in normal limbs. The difference obtained should correspond to the counter pressure and therefore to the appropriate class of compression.

The modern haemodynamic evaluation of CVI and VLL is mainly based on duplex ultrasound (DUS) and advanced technological imaging. ${ }^{13}$ Further haemodynamic investigations, as VPM and plethysmography, are systematically performed only in few specialized Centers. ${ }^{16-18}$ The majority of the Authors do not consider the invasive VPM as an essential systematic clinical investigation, ${ }^{11,12}$ nor they trust in Doppler VPM. Both the methods are not recommended in the International Guidelines for the Diagnosis and Treatment of Venous Disease. ${ }^{2-4}$

While the invasive VPM correspond to hydrostatic pressure in $\mathrm{mmHg}$, it must be supposed that many different anatomical, functional, mechanical and/or environmental factors of variation, most of them still unknown,
Correspondence: Daniele Pontello, via Damiano Chiesa 22, 33038 San Daniele del Friuli (UD), Italy.

Mobile. +39.328 .3266613 .

E-mail: ciuto77@virgilio.it

Key words: venous, pressure, elastic, compression, limbs.

Acknowledgments: this study was supported by CZ Medicali, Cuggiorno (MI), Italy.

Received for publication: 5 January 2014. Revision received: 23 August 2014.

Accepted for publication: 26 August 2014

This work is licensed under a Creative Commons Attribution 3.0 License (by-nc 3.0)

(C) Copyright L. Corcos et al., 2015

Licensee PAGEPress, Italy

Veins and Lymphatics 2015; 4:2230

doi:10.4081/vl.2015.2230

can influence the Doppler VPM, therefore the value resulting will be different by the hydrostatic one and should be better defined as VPI.

Since 1996 we introduced into our Vascular Laboratory the use of a new phlebologic software for the informatic filing of all the cases studied which has been presented in 1999. ${ }^{19}$ The emerging data and the experience acquired clearly demonstrated that the VPI measurements can give useful information concerning the severity of CVI, it is pathophysiology and the therapeutic implications. During our experience we applied the above-mentioned principles for the choice of the GES for the ECT of CVI and/or VVLL and we observed a relevant decrease of unsatisfied patients.

\section{Aims}

The aim of this study was to verify the value of VPI measurement in the choice of GES compression class and the preliminary results of ECT chosen on these bases.

\section{Materials and Methods}

In the period comprised between 1996 and 2002, 2098 LL of 1049 patients affected with CVI and/or other pathologies of the LL were studied by clinical and DUS (Sonoscape $1.000^{\circledR}$; SonoMed Bio, Inc., Bayamon, Puerto Rico) investigations and the time of reflux (R) was determined. The $\mathrm{R}$ was considered pathological when the time of duration was more than 1 second. ${ }^{19-21}$

In 1212 LL of 887 patients with CVI and 162 free from CVI, which were observed in our vascular laboratory by DUS examination, the VPI measurements were systematically performed. 
Limbs affected with venous malformations, deep venous thrombosis, superficial phlebitis were excluded. The VPI were detected by Doppler method (Dop 2.000 ${ }^{\circledR}$; Cardioline Milano, Italy) in standing position and after 10 tip-toeing exercises (ambulatory VPI) and the differential values were calculated. ${ }^{14,15,19}$ Patients affected with deep venous insufficiency were also examined in the same period and by the same investigations, but owing to the small number they were not included in the study. The distribution of the cases is reported in Table 1.

The VPI measurement is performed as follows. The patient is placed over a platform with banister in order to facilitate the standing position and the tip-toeing exercise. A pneumatic cuff of a sphygmomanometer is located at the middle calf and fixed with plaster. A Doppler 8 $\mathrm{MHz}$ flat or cylindrical probe is placed at the ankle level on the skin above the great saphenous vein (GSV), the small saphenous vein (SSV) and the posterior tibial (PTV). The patient is kept in standing position, the cuff is inflated up to $120 \mathrm{mmHg}$. The venous sound that can be heard during the cuff deflation and the VPI expressed in mmHg. corresponding to the reapperance of that sound is recorded. The same measurement is performed three times during the diagnostic session in order to verify the reproducibility of the values. ${ }^{15}$ Now the patient is asked to perform 10 tip-toe movements by lifting the body on the forward feet, one every second. The inflating-deflating and Doppler detection maneuvers are repeated according with the previous description upon the three districtis: GSV, SSV, PTV. All the data were collected by the phlebologic software. ${ }^{19}$ The Clinical-EtiologyAnatomy-Pathophysiology (CEAP) of every single limb was automatically calculated by the software and the $\mathrm{C}$ of CEAP was correlated with the site of reflux and the mean VPI values.

The mean values obtained were analyzed by Student test and standard deviation. The correlations of the VPI values with the $\mathrm{C}$ of CEAP were analyzed by the Bayesian test.

The mean difference of the standing VPI detected after 3 separate measurements in the GSV, SSV and PTV was calculated and compared with the mean normal values. In the patients with only one limb suffering with VVLL and/or CVI the comparison was done with the normal limb. A linear correlation between the differences of the VPI of the examined limbs compared with the mean normal values indicated the value of the counter pressure to be applied expressed in mmHg. On these bases the corresponding class of compression of the GES was chosen for every single limb (Figure 1). The proper size of the GES was carefully measured in the limb of every patient. A simple questionnaire was submitted to 96 patients affected with CVI/VVLL (from C2 to C6) which were previously subjected to the above described selection for
ECT in order to know how GES were tolerated after 60 days. The main questions were the following: i) Did you suffer with symptoms from CVI? ii) Did the application of GES improve your symptoms? iii) Did you suffer with pain or discomfort from GES?

\section{Results}

The mean VPI values of limbs affected with extended reflux in the whole GSV or limited to the leg were significantly higher (mean: 90 mmHg; range 67-113) than the ones detected in normal limbs (mean normal value: 77 mmHg; range 44-108) $(\mathrm{P}<0.01)$. The standing VPI in the GSV measured in the limbs with incompetent PVs were significantly higher (mean $96 \mathrm{mmHg}$; range 76-116) in comparison with the ones free from PVs incompetence (mean $84 \mathrm{mmHg}$; range 58-109) $(\mathrm{P}<0.01)$.

The mean VPI values in the PTV of limbs affected with extensive reflux in the GSV were significantly higher (mean $76 \mathrm{mmHg}$; range 54-97) than in normal limbs (mean normal value: $71 \mathrm{mmHg}$; range $43-99)(\mathrm{P}<0.01)$. The mean VPI values appeared to be significantly higher in the cases with incompetent PVs, with or without segmental GSV reflux (mean $81 \mathrm{mmHg}$; range 61-100) $(\mathrm{P}<0.01)$. In these cases the segmental GSV reflux was not related with increased VPI into the PTV.

The mean values of VPI measured into the incompetent SSV were higher of the 58.3\% (mean $71 \mathrm{~mm} \mathrm{Hg}$; range 46-100) if compared with normal limbs (mean normal value: 47
mmHg; range 16-82) $(\mathrm{P}<0.01)$ while the combination with PVs incompetence did not change the values.

All the mentioned districts were subjected to the ambulatory VPI measurements in every single limb and the differential pressure gradient, corresponding to the difference between VPI in standing position and after exercise, was calculated. No significant variation of the differential VPI in the various GSV/SSV segments and conditions, with or without PV incompetence, was found, except for the combination of GSV and PV incompetence. In these cases the differential VPI in the PTV was significantly higher (mean $30 \mathrm{mmHg}$; range 1446) than in the normal limbs (mean normal values $26 \mathrm{mmHg}$; range 26-41) $(\mathrm{P}<0.01)$.

Table 1. Distribution of the cases affected with chronic venous insufficiency/varicose veins of the lower limbs and subjected to the Doppler venous pressure index measurements.

\begin{tabular}{lc} 
Parameter & Value \\
No. patients examined & 1049 \\
No. patients without CVI & 162 \\
\hline No. patients with CVI & 887 \\
Average age & $52.4 \pm 16.2$ \\
\hline Male & $26 \%$ \\
Female & $74 \%$ \\
\hline Right & 554 \\
Left & 74 \\
\hline
\end{tabular}

CVI, chronic venous insufficiency.

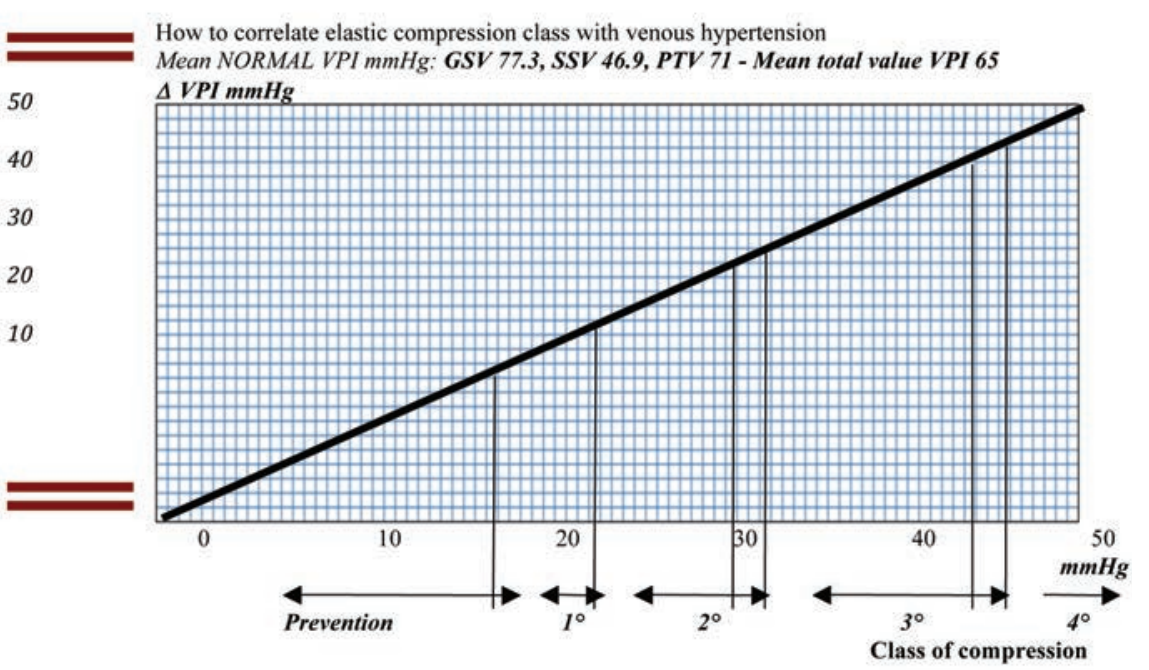

Figure 1. Criteria for the selection of the counter pressure of graduated elastic compression stockings necessary in the different clinical classes related to the CEAP classification, and venous pressure index (VPI). A linear correlation can be drawn. GSV, great saphenous vein; SSV, small saphenous vein; PTV, posterior tibial vein. 
All the data were analyzed by the Student's $t$ test.

The distribution of the VPI in the various districts of pathological and normal limbs were compared and analyzed by the Bayesian test. In the GSV a low prevalence (0.48), a high specificity (0.99) combined with high negative predictive value (0.88) were found. In the SSV the prevalence was 0.77 , while specificity and the negative predictive value were high (1.00). So it was also observed into the PTV (prevalence 0.69 , specificity 0.98 , negative predictive value 0.92 ).

The severity of the disease classified by $\mathrm{C}$ of CEAP appeared to be strictly related with the mean VPI measured in standing position in all the districts. However it must be noted that the range of variation measured in the limbs of the different $\mathrm{C}$ classes comprised a small number of cases characterized by quite low or high pressure which certainly required different classes of GES compression The data were examined by Student's test $(\mathrm{P}<0.01)$ (Figures 2-4).

The mean value of the standing VPI, which appeared to be the most significant measurement, observed in the 162 normal subjects the GSV's, SSV's and PTV's corresponded to the mean value of $65 \mathrm{mmHg}$ (range 37.6-93). Such values were used further on for a comparison with the ones detected in every single patient for clinical purposes. The differences observed were taken into consideration as they should correspond to the counter pressure to be applied for the ECT of CVI and/or VVLL. The counter pressure needed by every single limb was calculated on the basis of the linear correlation between the different pressure gradient and the pressure of the GES related with the class of compression (Figure 1).

Few patients of the whole casuistry, none belonging to the group of 96 who answered to the questionnaire, complained with pain or severe discomfort form GES. Three cases over the $96(3.12 \%)$ developed an allergic intolerance to the GES tissue after few days and interrupted the treatment. Six patients (6.25\%) which have been wearing the GES mainly suffered with burning leg/s owing to the eastern period of the treatment but kept on wearing GES and improved their symptoms from CVI. Ten elder patients, some of them affected with combined skeletal and/or joints pathology (10.4\%), encountered difficulties in wearing the GES, did not wear them continuously and did not improve. Eighty-one over the 83 who have been wearing GES (97.5\%) were satisfied for the total or partial relapse of the symptoms from CVI and did not complain with discomfort or pain from GES. Two C6 cases shown a relevant improvement of the healing process of ulcers and one was completely healed. The most frequent classes of compression applied were the $2^{\text {nd }}(\mathrm{N} 46=47.7 \%)$ and the $1^{\text {st }}(\mathrm{N}$ $33=34.3 \%$ ), while the so called preventive GES of less than $18 \mathrm{mmHg}$ were prescribed in 15 cases (15.6\%) and the $3 \mathrm{~d}$ class in 2 cases only (2.08\%). A VPI gradient which could indicate the application of GES of the $4^{\text {th }}$ class has never been found in this group.

\section{Discussion}

ECT is a fundamental primary or complementary method for reducing symptoms from CVI and VLL, for preventing superficial and deep venous thrombosis, for the conservative treatment of chronic venous ulcers and for reducing the worsening trend of venous disease, ulcers and complications. ${ }^{1-9}$ It is well known that in the clinical practice it is sometimes impossible to obtain full cooperation from patients who refuse the use of GES, because of the reasons exposed in the introduction of this paper. This inconvenient is more frequent that the persistence of symptoms from CVI due to a too low counter pressure applied. The present study seems to confirm on one hand the importance of the VPI measurements in phlebologic patients, on the other hand the advantages obtained by the appropriate choice of the GES class of compression on the basis of physical parameters and not simply of clinical subjective and objective criteria. ${ }^{1-9}$

Many efforts were done in the past and in the present for correctly defining the concept of CVI of the LL. Many descriptions of the pathophysiological mechanisms which lead to CVI were also discussed and one of them was venous hypertension. However no indications for a systematic haemodynamic instrumental evaluation nor any statistically analyzed data can be found in the literature except for the noninvasive Doppler method ${ }^{13-15,19}$ and photoplethysmography performed in specialized centers. ${ }^{16-19}$

Noninvasive VPI measurement can be proposed in the daily practice without encountering any difficulty from patients, who need a second leve $\mathrm{l}^{16}$ vascular diagnostics that we perform by noninvasive investigations: ${ }^{19}$ a detailed DUS examination, VPI measurement and, when necessary, photo-plethysmography. This approach made possible to have full cooperation from our patients and acquire a large experience which has shown how useful is a complete morphologic and functional information concerning the pathophysiological condition of every single case. A further advantage of the non-invasive Doppler VPI measurement is represented by information concerning the GSV, SSV and PTV districts separately, which can furnish interesting data concerning the balance between the superficial and deep venous function. ${ }^{13-15,19}$ Such detailed examination is not allowed by the invasive methods. ${ }^{11,12,16}$

The results of the study demonstrate the big advantage of taking significant data from the VPI examinations performed only in standing position and thus avoiding the dynamic evaluation, which is difficult especially for the elder patients or others who are affected with combined skeletal and/or joints pathology. The ambulatory VPI measurements were helpful in the few cases affected with deep CVI and occlusive venous disease, however they could not be

\section{VPI and Class "C" CEAP}

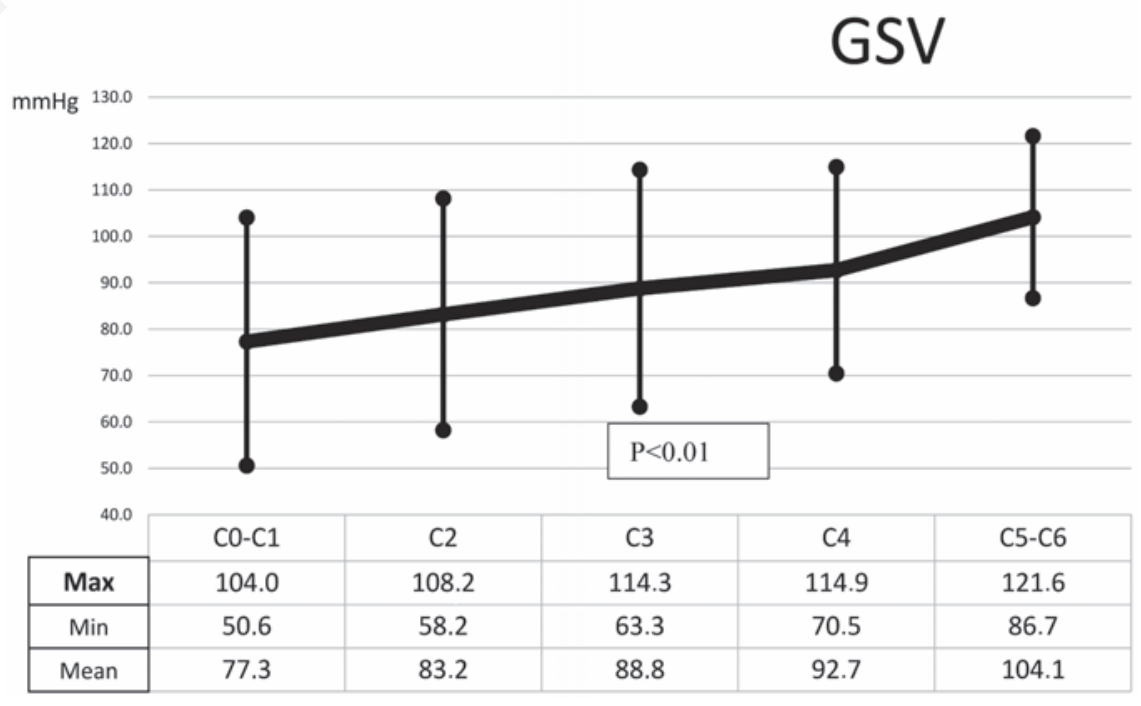

Figure 2. Correlation between the standing venous pressure index (VPI) of the great saphenous vein (GSV) and the C (of CEAP classification) class of severity. Statistical analysis performed by Student's test. 
taken into consideration owing to the low number of cases. Such dynamic measurements in combination with photoplethysmography seemed to give some useful data in patients affected with superficial CVI/VVLL and skeletal and/or joint's pathology. These addi- tional findings were not analyzed in the present work.

Until the statistical analysis of our data were completed we used to compare with the mean normal values of both the standing and post-exercise (ambulatory) VPI. It has been

\section{VPI and Class "C" CEAP}

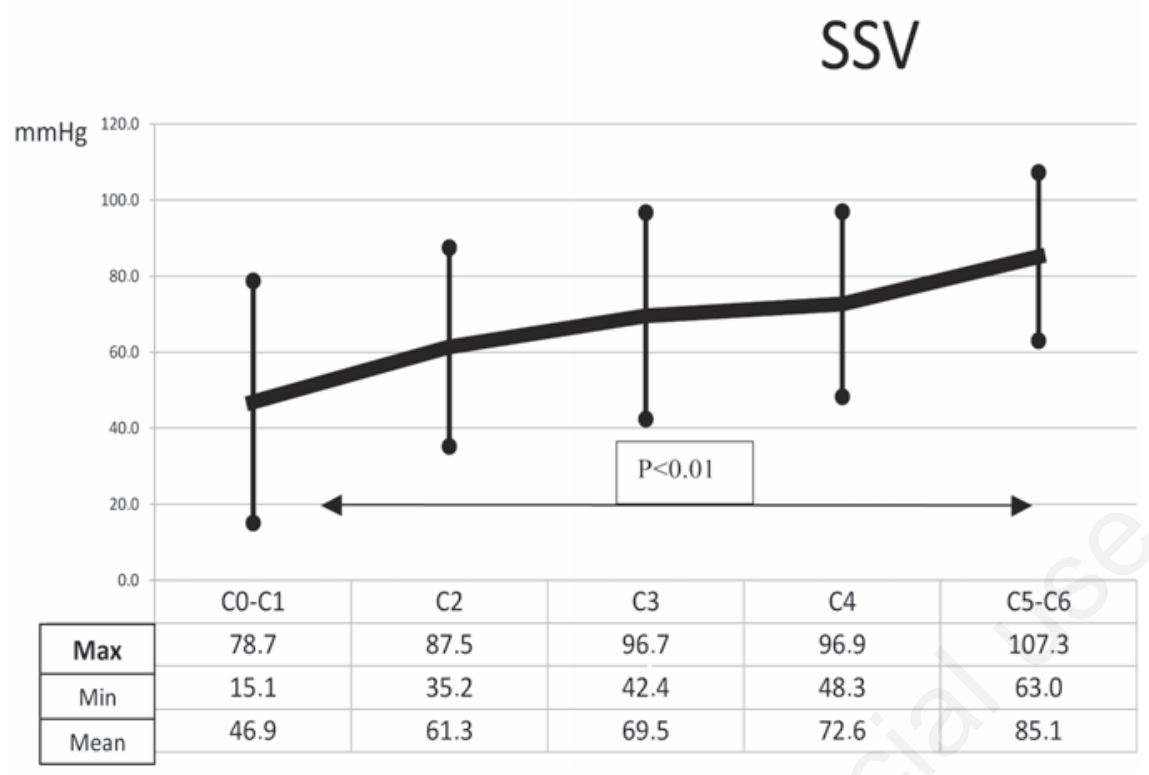

Figure 3. Correlation between the standing venous pressure index (VPI) of the small saphenous vein (SSV) and the C (of CEAP classification) class of severity. Statistical analysis performed by Student's test.

\section{VPI and Class "C" CEAP}

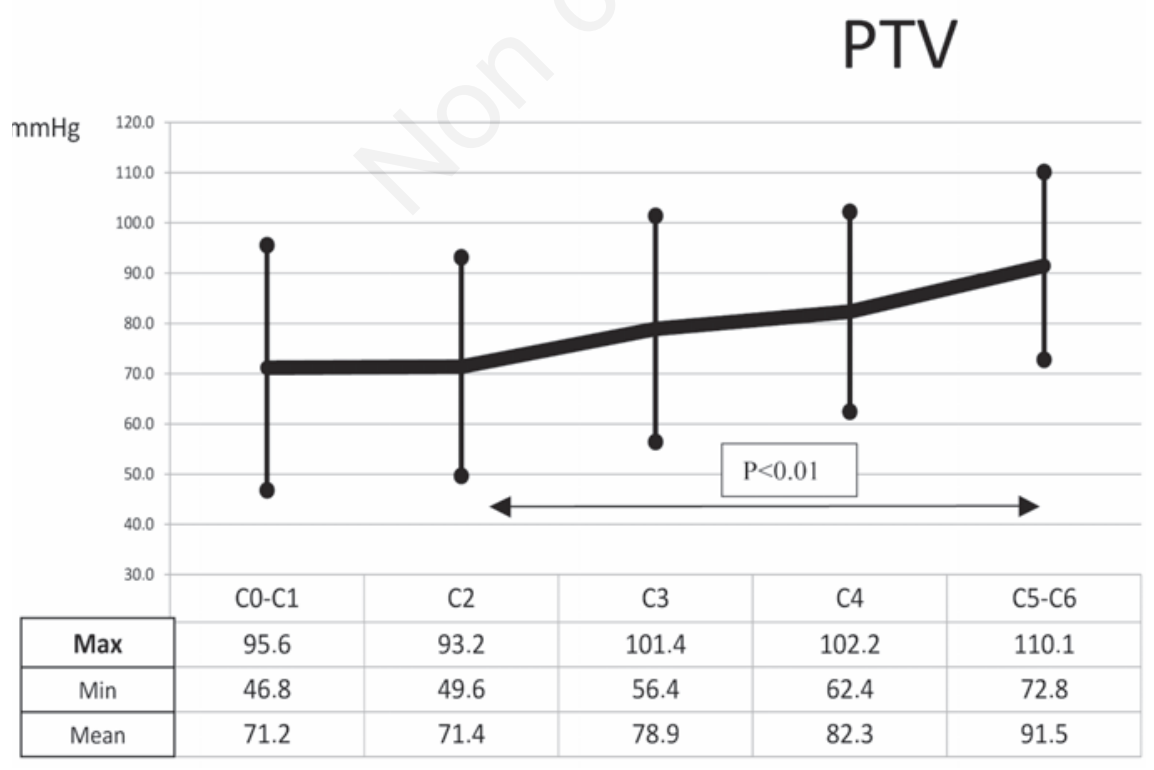

Figure 4. Correlation between the standing venous pressure index (VPI) of the posterior tibial vein (PTV) and the C (of CEAP classification) class of severity. Statistical analysis performed by Student's test.

always a complex calculation and very often it left some perplexities concerning the indication to GES. Since we understood that only the VPI measured in standing position was statistically significant in the evaluation of superficial CVI/WVLL, the calculation of the counter pressure gradient to be applied became more simple and the clinical results improved. During our previous experience, which is still in progress, many advantages in the prophylactic and therapeutic project of every single patient were found. The extension and surgical strategies can be more precisely planned. In cases affected with early superficial venous disease, with initial reflux and limited varicose veins, for those who are affected with CVI and/or VVLL but, in spite of our suggestions, refuse or have absolute exclusion criteria for any kind of invasive treatment, the VPI measurement can also represent a useful parameter for monitoring the progression of the disease, while the patient is subjected to conservative treatments. It can be easily affirmed that the more proper indication for ECT and prevention of CVI and/or VVLL can be found by the simple evaluation of the counter pressure necessary in every single case. Our experience demonstrates that only few patients of the whole casuistry and only $2 / 83$ (2.5\%) patients who have been wearing GES for 60 days complained with disadvantage or pain, when selected by the method above described. Allergies from elastic tissues and intolerability by the eastern climate are well known side effects of ECT and do not appear significant in the evaluation of the results; so do the difficulties encountered in wearing the GES mainly by the elder patients and those affected with various kinds of skeletal and joints infirmities. In addition to the demonstration of the increased tolerability of GES in patients selected by means of VPI measurements some other new information emerged from this work. On one hand we found a significant relation between the mean VPI and the C of CEAP of the three venous districts examined. This correlation indicates that the clinical criteria for deciding the appropriate GES compression class is still advisable. On the other hand it must be noted that the range of variation measured in the limbs of the different $\mathrm{C}$ classes comprised a small number of cases characterized by too low or too high pressure which certainly did not correspond to the simple clinical criteria and required different classes of GES compression in order to be effective and for avoiding the side effects from GES. It is possible to detect high VPI in cases affected with C2 and C3 VVLL and some low pressure in the more severe $\mathrm{C}$ (Figures 2-4); the indication to class 2 GES treatment appears to be more frequent than expected if compared with simple clinical criteria. The study performed and the results observed clearly demonstrate that discrepancies 
between the clinical class of CEAP and venous hypertension are to be expected in every single limb and this should be the main cause for some inappropriate indications of the GES compression class followed by discomfort of patients. We feel that the low number of the cases studied does not represent striking scientific documented evidence, but it can be easily verified by further research. The only disadvantage observed is that the VPI measurement by Doppler method is the high operator-dependency and the need for a learning period.

\section{Conclusions}

VPI measurements can represent the basis for a more precise selection of patients affected with venous disease of the lower limbs. Standing VPI is the most significant evaluation characterized by a high specificity and negative predictive value, it is the expression of the peripheral result of the valvular function in the various venous districts and it can be performed by a simple procedure. The Doppler VPI measurement is a highly predictive diagnostic and prognostic investigation and gives a great help for monitoring and treating all the patients affected with CVI and VVLL. R in GSV at the leg and in incompetent PV increases VPI and the severity of the disease. Extensive $R$ into GSV and PV can lead to secondary deep venous hypertension and it can be interpreted as an overload syndrome.

The above described experience seems to demonstrate that the best and selective choice of the GES can be performed on pathophysiological bases rather than on simple clinical criteria, which some times do not exactly correspond to the functional condition of the diseased limb. The clinical results of conservative physical treatment of superficial CVI and VVLL seem to be potentially improved by the choice of GES on the bases of VPI measurements.

\section{References}

1. Bassi G, Stemmer R. Traitements mecaniques fonctionelles en phlebolgie. Padova: Ed. Piccin; 1983. pp 67-153.

2. Stemmer R. Theoretical and practical bases of compression. J Mal Vasc 1992;17: 329-32.

3. Italian College of Phlebology. Guidelines on compression therapy. Acta Phlebologica 2000;1:11-6.

4. Collegio Italiano di Flebologia. Linee guida diagnostico terapeutiche delle malattie delle vene e dei linfatici. Acta Phlebologica 2003;4:14:10-2.

5. O'Meara S, Cullum N, Nelson EA, Dumville JC. Compression for venous leg ulcers. Cochrane Database Syst Rev 2012;11: CD000265.

6. Partsch H, Flour M, Smith PC. International Compression Club. Indications for compression therapy in venous and lymphatic disease consensus based on experimental data and scientific evidence. Under the auspices of the IUP. Int Angiol 2008;27:193-219.

7. Palfreyman SJ, Michaels JA. A systematic review of compression hosiery for uncomplicated varicose veins. Phlebology 2009; 24:13-33.

8. Cataldo JL, de Godoy JM, de Barros N. The use of compression stockings for venous disorders in Brazil. Phlebology 2012;27:33-7.

9. Lattimer CR, Azzam M, Kalodiki E, et al. Compression stockings significantly improve hemodynamic performance in post-thrombotic syndrome irrespective of class or length. J Vasc Surg. 2013;58:158-65.
10. Hojensgard IC, Stürup H. Static and dynamic pressures in superficial and deep vein of the lower extremity in man. Acta Phys. Scandinav 1952;27:49-67.

11. Neglen P, Raju S. Ambulatory venous pressure revisited. J Vasc Surg. 2000;31:1206-13.

12. Meissner MH, Moneta G, Burnand K, et al. The hemodynamics and diagnosis of venous disease. J Vasc Surg 2007;46:4S-24S.

13. Gayliss H. Some observations on peripheral venous pressure using a non-invasive technique: a preliminary report. Br J Surg 1975;62:259-63.

14. Bartolo M. Phlebodopplertensiometry, a non-invasive method for measuring venous pressure. Folia Angiol 1977;25:199-203.

15. Bartolo M, Nicosia PR, Antignani PL, et al. Noninvasive venous pressure measurements in different venous diseases. Angiology 1983;34:717-23.

16. Nicolaides AN. Investigation of Chronic venous insufficiency: a consensus statement. Circulation 2000;102:e126-63.

17. Fronek A. Noninvasive diagnostics in vascular disease. New York: McGraw-Hill Book; 1989. pp 11-85.

18. Schultz-Ehremburg U, Blazek V, Fronek A, et al. Venous photophlethysmography and invasive venous pressure measurement - a multicentric comparative study. Part 2: results. In Advances in computer-aided noninvasive vascular diagnostics. Proceedings of the $9^{\text {th }}$ International ymposium CNVD 2000. January 21-23, 2000. Bratislava, Dusseldorf: VDI-Verlag GmbH; 1994. pp 45-52.

19. Spina T, Corcos L, Peruzzi G, et al. The value of Doppler venous pressure index in the diagnosis of chronic venous insufficiency of the lower limbs. Int Angiol 2009;28:92. 